\title{
A soft tissue groin mass
}

\author{
Harun Gupta $•$ Andrew J. Grainger
}

Published online: 13 March 2010

(C) ISS 2010

\section{Groin lump in a pregnant woman}

Diagnosis

Varicosities of the round ligament

\section{Discussion}

Round ligament varicosities are engorged pelvic veins, that have extended into the inguinal canal around the round ligament. These typically occur during and after the second trimester of pregnancy and present as soft and non-tender masses in the inguinal region.

The most common differential diagnosis of a groin lump with this presentation is an inguinal hernia [1-4]. Although inguinal hernias are uncommon in women, increased intraabdominal pressure during pregnancy can lead to this condition. Its incidence in pregnancy has been reported to be 1 in 1,000-3,000 [5]. Distinguishing round ligament varicosities from inguinal hernia on clinical grounds can be difficult and is often not possible as both have a similar location, can be reducible or irreducible and can transmit

The case presentation can be found at doi:10.1007/s00256-010-0885-5.

H. Gupta $\cdot$ A. J. Grainger

Department of Musculoskeletal Radiology,

Leeds Teaching Hospitals,

Leeds, UK

\section{A. J. Grainger $(\varangle)$}

Department of MSK Radiology,

Chapel Allerton Orthopaedic Centre,

Leeds LS7 4SA, UK

e-mail: andrew.grainger@leedsth.nhs.uk cough impulses [1-4]. Other possible causes of an inguinal mass include femoral hernia, persistent embryonic remnants (cyst of the canal of Nuck), lymphadenopathy, soft tissue tumour and abscess formation.

In this case the history of sudden onset in the third trimester of pregnancy suggested the diagnosis, but the correct diagnosis was confirmed by the typical appearances on Doppler ultrasound [6]. Many of the other possible diagnoses, such as lymphadenopathy, hernias, cysts and abscess, also have characteristic ultrasound appearances, while the absence of a solid component to the mass would make soft tissue tumour unlikely. Round ligament varicosities appear as large dilated spaces on ultrasound, located within the inguinal canal and passing through the deep inguinal ring (lateral to the inferior epigastric vessels). In our case the varicosities also passed through the superficial ring. Colour or power Doppler confirms the presence of blood flow within the veins comprising the varicocoele, and this characteristically augments with the Valsalva manoeuvre [7]. The inferior epigastric vessels are easily seen on ultrasound and lie immediately medial to the deep inguinal ring. As with investigation of inguinal hernia, they provide a landmark for the sonographer. The superficial inguinal ring lies immediately lateral to the pubic tubercle, which provides a useful bony landmark. Although the veins are associated with the round ligament of the uterus, the ligament itself was not visualised. In the normal female inguinal canal, the veins and artery characteristically mark the course of the round ligament on ultrasound.

Round ligament varicosities are an accentuation of a physiological process and correct diagnosis is imperative to avoid unnecessary surgery. They are managed conservatively and resolve in the post-partum period. The resolution post-partum helps to exclude other causes of vascular mass, such as an arterial-venous malformation, although the 
sudden onset during pregnancy and absence of arterial flow also make this diagnosis unlikely.

There are many cases in the literature where surgery for suspected hernia has resulted in round ligament varicosities being diagnosed intra-operatively [8]. Ultrasound reliably makes the diagnosis and avoids such unnecessary surgery.

\section{References}

1. Tomkinson JS, Winterton WR. Varicoceles of the round ligament in pregnancy, simulating inguinal herniae. Br Med J. 1955;1:889-90.

2. Guillem P, Bounoua F, Duval G. Round ligament varicosities mimicking inguinal hernia: a diagnostic challenge during pregnancy. Acta Chir Belg. 2001;101:310-1.
3. Chi C, Taylor A, Munjuluri N, Abdul-Kadir R. A diagnostic dilemma: round ligament varicosities in pregnancy. Acta Obstet Gynecol Scand. 2005;84:1126-7.

4. Pilkington SA, Rees M, Jones O, Green I. Ultrasound diagnosis of round ligament varicosities mimicking inguinal hernias in pregnancy. Ann R Coll Surg Engl. 2004;86:400.

5. Barber HRK, Garber EA. Editorial comments on Kesseler HJ. In: Barber HRK, Grabe EA, editors. Hernias in pregnancy, surgical disease in pregnancy. Philadelphia: Saunders; 1974. p. 165.

6. Cheng D, Lam H, Lam C. Round ligament varices in pregnancy mimicking inguinal hernia: an ultrasound diagnosis. Ultrasound Obstet Gynecol. 1997;9:198-9.

7. Nguyen QH, Gruenewald SM. Doppler sonography in the diagnosis of round ligament varicosities during pregnancy. J Clin Ultrasound. 2007;36(3):177-9.

8. Ijpma FFA, Boddeus KM, de Haan HH, van Geldere D. Bilateral round ligament varicosities mimicking inguinal hernia during pregnancy. Hernia. 2009;13:85-8. 